\title{
Prediction and Experimental Evidence for Thermodynamically Stable Charged Orbital Domain Walls
}

\author{
Qing'an Li, ${ }^{1,}$ K. E. Gray, ${ }^{1}$ S. B. Wilkins, ${ }^{2}$ M. Garcia Fernandez, ${ }^{2}$ S. Rosenkranz, ${ }^{1}$ H. Zheng, ${ }^{1}$ and J. F. Mitchell ${ }^{1}$ \\ ${ }^{1}$ Materials Science Division, Argonne National Laboratory, Argonne, Illinois 60439, USA \\ ${ }^{2}$ Condensed Matter Physics and Materials Science Department, \\ Brookhaven National Laboratory, Upton, New York 11973, USA
}

(Received 21 March 2014; revised manuscript received 18 June 2014; published 18 August 2014)

On theoretical grounds, we show that orbital domain walls (ODWs), which are known to exist in the charge and orbital ordered layered manganite $\mathrm{LaSr}_{2} \mathrm{Mn}_{2} \mathrm{O}_{7}$, should be partially charged as a result of competition between orbital-induced strain and Coulomb repulsion. This unexpected result provides the necessary condition for the known thermodynamic stability of these ODWs, which are unlike the more typical domain walls that arise only from an external field. We offer experimental data consistent with this theoretical framework through a combined transport and x-ray-diffraction study. In particular, our transport data on this charge and orbital ordered manganite exhibit abrupt transformations to higher conductance at a threshold electric field. As transport phenomena closely resemble effects found for sliding charge-density waves (SCDWs) in pseudo-one-dimensional (1D) materials, a SCDW along such pseudo-1D ODWs provides a natural explanation of our data. Importantly, x-ray-diffraction data eliminate heating and melting of charge order as tenable alternative explanations of our data.

DOI: 10.1103/PhysRevX.4.031028

\section{INTRODUCTION}

As shown by the discovery of superconductivity at the boundary between $\mathrm{SrTiO}_{3}$ and $\mathrm{LaAlO}_{3}$ in oxide heterostructures [1,2], interfaces reveal new phenomena when charges transfer onto them from their surroundings. Such interfacial behavior provides both scientific and technological opportunities, as the reduced dimensionality makes it appealing for nanoscale devices. Among the myriad types of interfaces, atomically homogeneous domain walls are special because their spontaneous creation, with charge transfer onto them, is a rare event [3]. Previous diffraction studies of charge and orbital ordered (COO) manganites $[4,5]$ revealed ordered plaquettes (length scales of several tens of $\mathrm{nm}$ [5]) that are bounded by atomically homogeneous orbital domain walls (ODWs); see, e.g., Fig. 1. Thus, the theoretical insight derived from the work presented here-that ODWs in COO manganites must be charged - adds a new member to this short list and provides a mechanism for their known thermodynamic stability.

To better understand what is special about these ODWs, we first observe that the generic pathway to domain structure is a positive interface (wall) energy that is

\footnotetext{
*qali@anl.gov
}

Published by the American Physical Society under the terms of the Creative Commons Attribution 3.0 License. Further distribution of this work must maintain attribution to the author(s) and the published article's title, journal citation, and DOI.
Subject Areas: Condensed Matter Physics, Nanophysics, Strongly Correlated Materials

counterbalanced by the lowering of energy of an associated external field. Consequently, such structures are not stable in the thermodynamic limit. Ferromagnets, type-I superconductors in an applied magnetic field, and ferroelectrics are prime examples of such domain structures. For the $\mathrm{COO}$ manganites, considerations of the elastic interactions among orbitals [6] lead to a positive wall energy (see details below) for all four ODW configurations suggested by Hill et al. [5]. However, as there is no obvious (elastic or other)

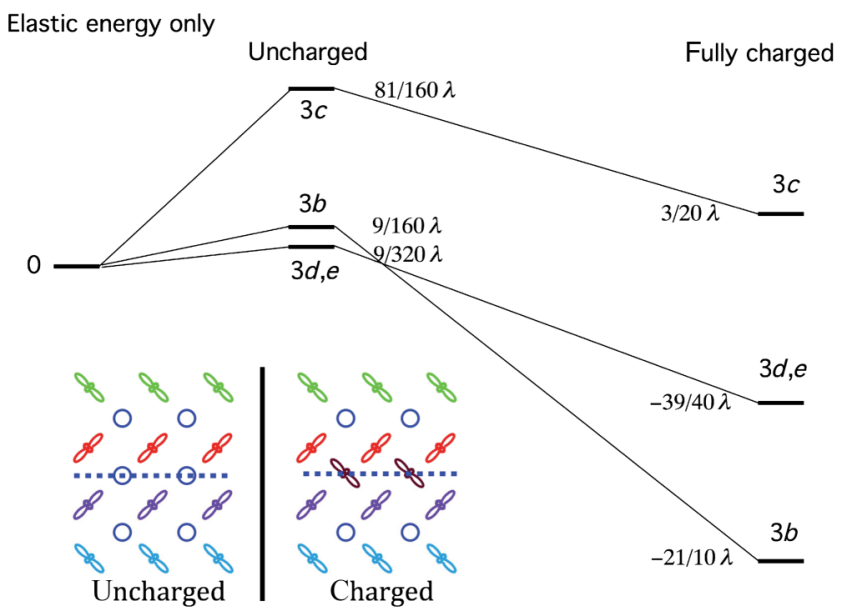

FIG. 1. The elastic energy levels of the configurations $3 b$ (shown as the inset), $3 c, 3 d$, and $3 e$ for uncharged and fully charged ODW, where $\lambda^{\prime} / \lambda=3 / 10$. The energy levels of fully charged ODW are reduced by a factor of 2.5 in the plot. 
external field to counterbalance the wall energy, these ODW configurations cannot achieve stability in the same way as the above examples. Here, we show instead that ODWs will be thermodynamically stable due to a process in which electrons are transferred onto a fraction of the $\mathrm{Mn}^{+4}$ sites along the ODWs shown in Ref. [5] from $\mathrm{Mn}^{+3}$ orbitals in the bulklike COO plaquettes (see a fully charged version in Fig. 1). Surprisingly, we find that for a sufficiently high density of added electrons, the strain energy, derived from orbital interactions [6], gives rise to a net negative elastic component to the domain-wall energy. It is only the compensating increase in Coulomb energy, due to the added electrons in the ODWs and holes left behind in the $\mathrm{COO}$ plaquettes, which prevents a total collapse of orbital order. Our detailed calculations confirm thermodynamic stability for a charge-density wave along the ODW with a periodicity of approximately 5 to 35 atomic sites.

\section{THEORETICAL MODEL}

In the previous x-ray-diffraction studies of ODWs in COO manganites, Hill et al. [5] have suggested that the materials consist of ordered COO plaquettes (length scales of several tens of nm [5]). These plaquettes are bounded by one-dimensional ODWs that are of four types, each of which has only $\mathrm{Mn}^{+4}$ sites along the ODW. (See the realspace orbital configurations labeled b-e in Fig. 3 of Ref. [5].) Considering the elastic interactions among orbitals [6], these four ODW types are shown to have energies per $\mathrm{Mn}^{+4}$ site of $\Delta E_{3 b}=9 \lambda / 32-3 \lambda^{\prime} / 4$ and $\Delta E_{3 c}=9 \lambda / 32+3 \lambda^{\prime} / 4$, while $\Delta E_{3 d}$ and $\Delta E_{3 e}$ are each one-half of $\Delta E_{3 b}$ [Eqs. (7)-(9) of Ref. [6]]. Here, the elastic interactions between orbitals are characterized [6] by $\lambda$ along the principal $a$ and $b$ axes and $\lambda^{\prime}$ for the $a b$-plane body diagonal. As Khomskii and Kugel [6] have pointed out, the stability criterion for the COO phase implies that $\lambda^{\prime} / \lambda<3 / 8$; thus, all four ODW configurations must have a positive energy of formation-and hence be thermodynamically unfavorable. Why, then, do they form?

We argue that they form by transferring electrons onto the $\mathrm{Mn}^{+4}$ sites of an ODW from the neighboring $\mathrm{COO}$ ordered plaquettes, a process which drastically alters the above picture: Then, the elastic energy for charged ODWs is favorable for $3 b, 3 d$, and $3 e$. Consider the elastic interactions among orbitals [6] for the most favorable charged ODW, i.e., the orbital labeled $3 b$, which consists of $\mathrm{Mn}^{+4}$ sites surrounded by four parallel $3 x^{2}-r^{2}$ (or $3 y^{2}-r^{2}$ ) nearest-neighbor orbitals (see Fig. 1). We find that placing electrons on those $\mathrm{Mn}^{+4}$ sites with $3 y^{2}-r^{2}$ (or $3 x^{2}-r^{2}$ ) orbitals, i.e., perpendicular to the four nearest-neighbor orbitals, lowers the energy per site by $2 \lambda$. Importantly, the lowering of the elastic energy by adding these electrons will more than compensate the elastic energy cost to create the complementary hole in the $\mathrm{COO}$ plaquette, which is $-\lambda^{\prime} / 6+\lambda / 4<3 \lambda / 16$ (since $\lambda^{\prime} / \lambda<3 / 8$ ). Similarly, for the other orbital configurations ( $3 d$ and $3 e$ ), we find that charging lowers the elastic energy. The resulting energies, shown in Fig. 1, assume a charge on every $\mathrm{Mn}^{+4}$ site and are calculated relative to the unperturbed $\mathrm{COO}$ state.

\section{CALCULATION OF COULOMB ENERGY AND CHARGE PERIODICITY}

Indeed, unchecked, this negative elastic energy would fill the crystal structure with domain walls and destroy long-range order. However, a simple mean-field model demonstrates that it is the compensation by the positive Coulomb-repulsion energy of the added charges that stabilizes a domain structure to retain the zigzag orbital order in the plaquettes. If we assume that the lowest-energy configuration is a periodic charge distribution along the ODW, the relevant Madelung constant $\alpha$ is readily calculated as 1.61556 [7]. To calculate elastic and Coulomb energies and compare them quantitatively, we need to determine several materials parameters; specifically, we need $\lambda$ and $\lambda^{\prime}$ for the elastic energy and the characteristic energy scale $E_{0}=q^{2} /\left(4 \pi \epsilon_{0} \epsilon_{r} r\right)$ for the Coulomb energy, where $r$ is the shortest distance between $\mathrm{Mn}^{3+}$ and $\mathrm{Mn}^{4+}$ sites, $\epsilon_{0}$ is electric permittivity in vacuum, $\epsilon_{r}$ is the dielectric constant of the material, and $q$ is the effective charge disproportionation expected to be approximately $0.2 e$ [8].

Although $E_{0}$ contains two parameters $q$ and $\epsilon_{r}$ in the present study, we only need the value of $E_{0}$. Fortunately, $E_{0}$ and $\lambda$ can be estimated from the orbital ordering (OO) and charge ordering (CO) critical temperatures $T_{\mathrm{OO}}$ and $T_{\mathrm{CO}}$, while $\lambda^{\prime}$ is constrained by the stability criterion of the COO state, namely, $0<\lambda^{\prime}<3 \lambda / 8$. For the elastic energy, we apply an Ising-type model as a means to establish the relationship between the materials parameter $\lambda$ and the orbital ordering transition temperature $T_{\mathrm{OO}}$ within meanfield theory. Others [9] have used this approach to describe orbital ordering in manganites, and their Hamiltonian is written as $H=J \sum_{i, j} \sigma_{i} \sigma_{j}+J^{\prime} \sum_{i, k} \sigma_{i} \sigma_{k}$, where $\sigma_{i}= \pm 1$ label orbitals on $\mathrm{Mn}^{3+}$ sites and $J$ and $J^{\prime}$ are the nearestneighbor and next-nearest-neighbor interactions, respectively. The elastic interaction energy [6] per $\mathrm{Mn}^{3+}$ from its four nearest neighbors (along the $x$ or $y$ directions) is $-\lambda / 4$, and from its four next-nearest neighbors is $\lambda^{\prime} / 6$ (diagonal direction) in the inset of Fig. 1. Therefore, we obtain $J=\lambda / 16$ and $J^{\prime}=-\lambda^{\prime} / 24$. Then, according to mean-field theory [10], $T_{\mathrm{OO}}=z_{1} J-z_{2} J^{\prime}=\lambda / 4-\lambda^{\prime} / 6$, where $z_{1}=$ $z_{2}=4$ are the number of nearest-neighbor and nextnearest-neighbor sites, respectively. Finally, we obtain $\lambda=4 T_{\mathrm{OO}} /\left(1-\left(2 \lambda^{\prime} / 3 \lambda\right)\right)$.

It is convenient to perform a similar transformation to parametrize the Coulomb interaction. The Hamiltonian of the nearest-neighbor Ising model for $\mathrm{CO}$ can be written as $H=J_{\mathrm{CO}} \sum_{i, j} \sigma_{i} \sigma_{j}$, where $\sigma_{i}= \pm 1$ represent hole and electron, respectively, and $J_{\mathrm{CO}}$ is the nearest-neighbor interaction. Similarly to the above example of the elastic energy, one can use this model to obtain a relation between 
$E_{0}$ for the Coulomb energy (above) and the $\mathrm{CO}$ transition temperature $T_{\mathrm{CO}}$. Because of the long-range nature of the Coulomb interaction, any electron or hole interacts with all other electrons and holes, so its Coulomb energy is $-\alpha E_{0}$. To simulate such long-range Coulomb interactions by the nearest-neighbor interaction $J_{\mathrm{CO}}$ needed for an Ising model, we can simply set $J_{\mathrm{CO}}=-\alpha E_{0} / z$, where $z$ is the number of nearest neighbors. Then, we obtain $E_{0}=$ $k_{B} T_{\mathrm{CO}} / \alpha$, since $T_{\mathrm{CO}}=z\left|J_{\mathrm{CO}}\right|$. From $E_{0}$, we estimate $\epsilon_{r} \approx$ 12 by using $q \approx 0.2 e$. This indirect determination of $\epsilon_{r}$ ignores the fact that it depends on the specific features of the orbital structure and is not well defined at the atomic scale. However, the $T_{\mathrm{CO}}$ value we use in $E_{0}$ is that found experimentally for the material with its $\mathrm{nm}$-scale domain structure, so one might expect that any issues with $\epsilon_{r}$ affecting the Coulomb energy in our domain-wall calculation would similarly affect $T_{\mathrm{CO}}$.

Experimentally, $T_{\mathrm{OO}}$ and $T_{\mathrm{CO}}$ are very close and not readily distinguishable, so that little error is introduced by assuming them equal to $T_{\mathrm{COO}} \approx 227 \mathrm{~K}$. The materials parameters $\lambda$ and $E_{0}$ can be obtained within this mean-field ansatz, with a correction to account for $\lambda^{\prime} / \lambda$.

The resulting charge-period dependence of the Coulomb energy, $E_{C}$, for electrons periodically replacing holes on an ODW is shown in Fig. 2. As expected intuitively, this electrostatic energy rises rapidly as $N$ decreases, implying that $N$ cannot be too small. Note that the result in Fig. 2 assumes that the removal of electrons from the "bulk" plaquettes can be calculated in the infinitely dilute limit, thus contributing $-(\alpha / 4)=0.40389$ per site. That is, the number of electrons removed from the plaquettes is a very small fraction in the total number of $\mathrm{Mn}^{3+}$ sites in the

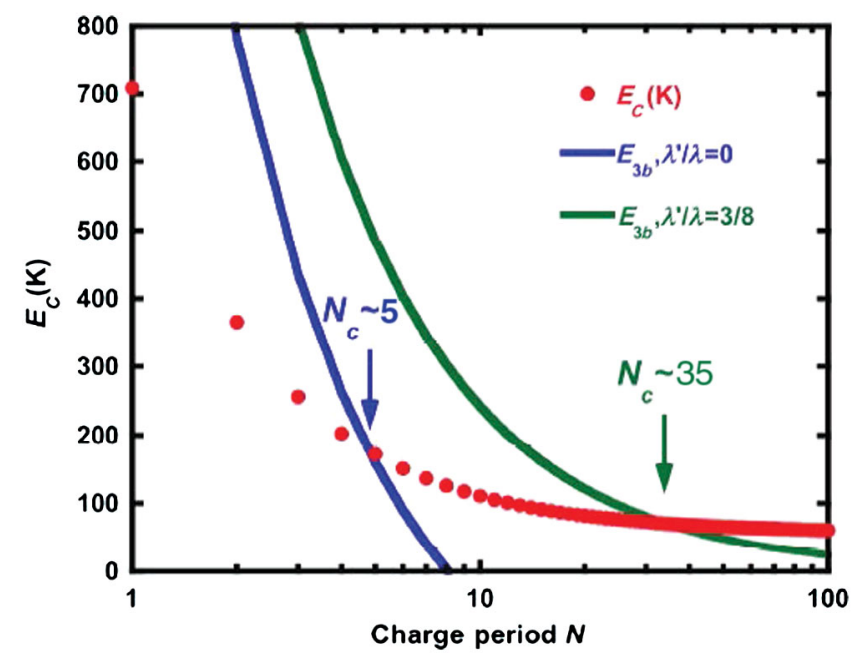

FIG. 2. Values of the Coulomb energy, $E_{C}$, and the absolute values of the negative elastic energies, $E_{3 b}$, have been averaged over the Mn sites along an ODW as a function of the charge period, $N$. The critical value of $N$, that occurs when these energies compensate, is $N_{c}$ and it is shown for theoretically allowed extremal values of $\lambda^{\prime} / \lambda=0$ and $3 / 8$. plaquettes. This constant term is very small and is not included in Fig. 2.

For finite $N$, only a fraction $1 / N$ of the $\mathrm{Mn}^{4+}$ sites on the ODW contributes to the elastic energy gain such that the average elastic energy gain is $\left[(N-1) \Delta E_{3 b}-2 \lambda\right] / N$, where $\Delta E_{3 b}=9 \lambda / 32-3 \lambda^{\prime} / 4$ comes from Ref. [6]. Then, even in the limit of the Coulomb energy going to 0 , there is an upper bound on $N$ equal to $1+64 /\left(9-24 \lambda^{\prime} / \lambda\right)$. That agrees with intuition that the added charges cannot be infinitesimally dilute. Since the average Coulomb energy decreases monotonically with $N$, there is a critical value of $N_{c}$ determined by competition between the elastic and Coulomb energies, as shown graphically in Fig. 2 for two $\lambda^{\prime} / \lambda$ values. These calculations indicate that the optimal $N_{c}$ for the $3 b$ configuration lies between approximately 5 and 35 for the theoretically allowed range [11] of $\lambda^{\prime}$ from 0 to 3 $3 \lambda / 8$, respectively. Since $\lambda^{\prime} / \lambda=3 / 8$ is the limit of stability of the COO lattice [6], one might expect that $N_{c}$ is not too close to 35 . Following the same procedure, we obtain the optimal $N_{c}$ for $3 d$ and $3 e$ to range between approximately 4 and 18. No solution is found for pattern $3 c$, suggesting this pattern is not a thermodynamically favorable one and may not exist in the layered manganites.

\section{EXPERIMENTAL TRANSPORT AND X-RAY DIFFRACTION}

Experimental evidence for partially charged ODWs comes from our nonequilibrium (NE) current-voltage data $I(V)$ measured in the $\mathrm{COO}$ bilayer manganite $\mathrm{LaSr}_{2} \mathrm{Mn}_{2} \mathrm{O}_{7}$ at high currents. These data exhibit abrupt transformations [see Fig. 3(a)] to higher conductance $I / V$ at a threshold voltage, while x-ray-diffraction data, discussed below, confirm that these $I(V)$ are neither caused by heating [12] nor melting of orbital order [13], as had been concluded in previous manganite experiments of others $[12,13]$. Our situation closely resembles traditional sliding charge-density-wave (SCDW) systems [14], including their pseudo-one-dimensional (1D) nature, their $I(V)$, and the threshold depinning voltage. Taken together, our theoretical insight and its natural explanation of our experimental data provide strong evidence for an unexpected phenomenon-a SCDW along the periodically charged members of an ODW network. Note that this SCDW picture is very different from that of Cox et al. [15], who suggested that it is the two-dimensional superstructure of the $\mathrm{COO}$ phase of $\mathrm{La}_{0.5} \mathrm{Ca}_{0.5} \mathrm{MnO}_{3}$ that slides in the presence of an electric field.

To obtain the data to support this scenario, crystals of $\mathrm{La}_{2-2 x} \mathrm{Sr}_{1+2 x} \mathrm{Mn}_{2} \mathrm{O}_{7}$ are melt grown using flowing $20 \% \mathrm{O}_{2}$ (mixed with $\mathrm{Ar}$ ) in an optical image floating-zone furnace and then cut into rectangular shapes with the $c$ axis oriented perpendicular to their largest flat surfaces. For transport measurements, four gold electrodes are deposited by dc sputtering onto each $c$-axis-normal surface [inset of Fig. 3(b)]. The two crystals used for our studies are selected 

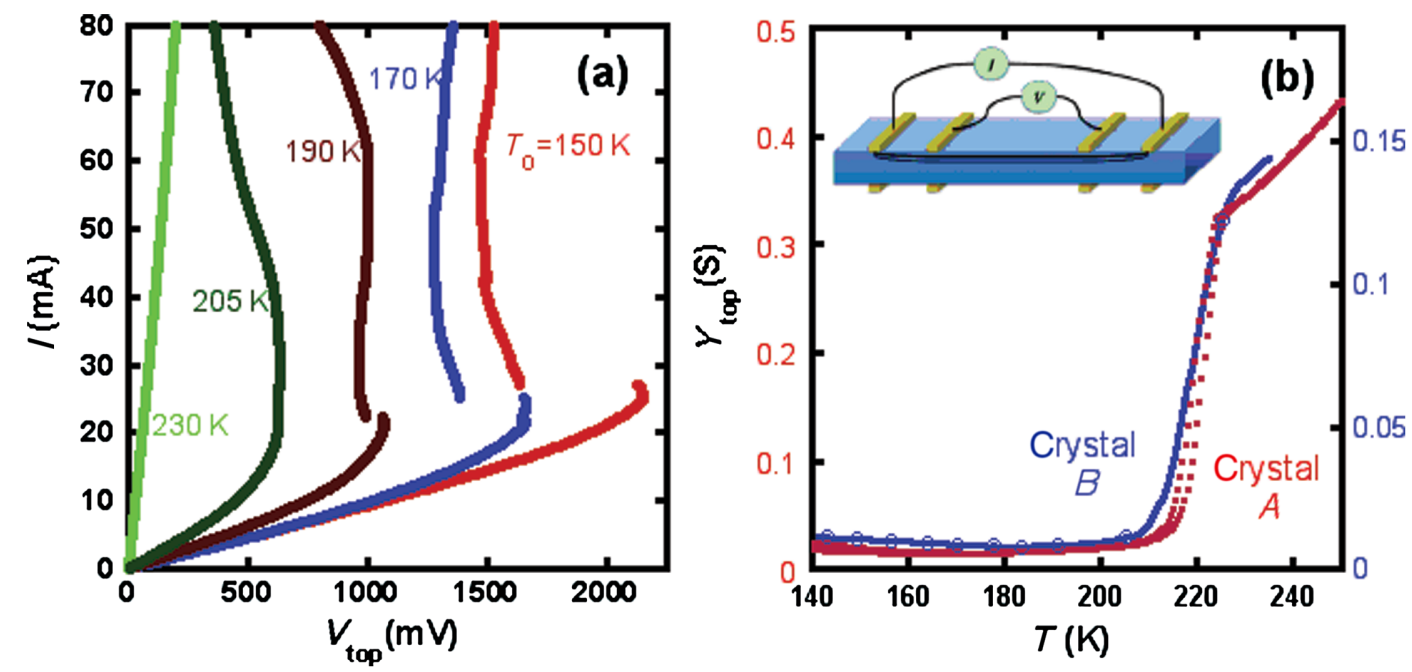

FIG. 3. (a) Current-voltage curves for crystal $A$ at various indicated bath temperatures. (b) Temperature dependence of conductance measured in the four-terminal method (inset) for crystals $A$ and $B$.

on the basis of their narrow $\Delta x$, i.e., the homogeneity of their $\mathrm{Sr}$ to La ratio, but each differs slightly from the nominal $x=0.5$. Their homogeneity is determined by permuting current and voltage leads among the eight electrodes [16] and measuring the conductance $Y$ at a small current of $0.1 \mathrm{~mA}$. Such data for all four configurations are within $10 \%$, thus placing them among the most homogeneous bilayer manganites we have ever investigated. One permutation is shown in Fig. 3(b) for crystals $A$ and $B$ as a function of temperature $T$. The $\mathrm{COO}$ (majority) phase exhibits a sharp transition to a high-temperature paramagnetic insulator at $T_{\mathrm{COO}} \approx 225 \mathrm{~K}$, while the slight rise in $Y(T)$ below $180 \mathrm{~K}$ reflects a small portion of each crystal, due to slight compositional $(x)$ inhomogeneity, which has a higher-conductivity, $A$-type antiferromagnetic ground state [17].

For our transport data, the current $I$ is injected through the outermost contacts and the voltage $V$ is measured across the innermost contacts of one (top) surface [see the inset of Fig. 3(b)]. This arrangement results in a large surface current density that falls off exponentially with distance from the top surface [18] with a characteristic length $D_{\text {eff }}=L / \pi \xi$, where $\xi=\sqrt{\left(\rho_{c} / \rho_{a b}\right)}$ is the temperaturedependent resistivity anisotropy and $L$ is the distance between current contacts. In our crystals, $D_{\text {eff }}$ is in the range of $20-40 \mu \mathrm{m}$.

The key experimental observation is the approximately vertical (constant-voltage) portion of the current-voltage curves $I(V)$ that appear in Fig. 3(a) after a clearly visible abrupt jump, along the current-bias load line of our experiment at approximately $20 \mathrm{~mA}$, to a higher-conductance, i.e., lower-voltage, state. For $T_{0}=205 \mathrm{~K}$, the abrupt jump is negligible but the qualitative behavior is otherwise identical. Note that the $T_{0}=230 \mathrm{~K}$ data are taken above the COO temperature $T_{\mathrm{COO}}$, and the $I(V)$ is approximately
Ohmic up to $80 \mathrm{~mA}$. For other $T_{0}$, the $I(V)$ are initially Ohmic with the conductance increasing smoothly with $I$ in a manner consistent with slight heating [based on the thermal equilibrium conductance $Y(T)$ shown in Fig. 3(b)]. At the highest currents, $V$ drops below the vertical $I(V)$ (e.g., at $T_{0}=190 \mathrm{~K}$ ) and approaches the Ohmic behavior for $T_{0}=230 \mathrm{~K}>T_{\mathrm{COO}}$

\section{HEATING EFFECTS}

It is the vertical portions of the $I(V)$, shown in Fig. 3(a) for our specimen labeled crystal $A$, that are of interest-we need to establish that this behavior is not simply and only the result of inevitable Joule heating or melting of the $\mathrm{COO}$ state. To address heating, we utilize both electrical transport and high-resolution synchrotron x-ray diffraction to provide calibrated thermometers within the crystal itself to determine an effective temperature $T^{*}$ when high currents are applied. These $T^{*}$ are inevitably greater than the bath temperature $T_{0}$. For transport, we use the four top contacts of crystal $A$ to obtain the high-dc-current conductance $Y_{\text {top }}(I)$, while the low-current-ac conductance measured by the four bottom contacts $Y_{\text {bot }}$ serves as a thermometer. Since the current penetration depth $D_{\text {eff }}=20 \mu \mathrm{m}$ for crystal $A$ is much less than the crystal thickness of $300 \mu \mathrm{m}$, these measurements are electrically isolated, and the results are easily visualized in the time dependencies. In Fig. 4, $Y_{\text {top }}$ and $Y_{\text {bot }}$ have been converted to $T_{\text {top }}^{*}$ and $T_{\text {bot }}^{*}$ by using the corresponding low-current $Y(T)$, such as Fig. 3(b), as calibrations. These data reveal simple heating up to $3 \mathrm{~s}$, with the larger $\Delta T_{\text {top }}^{*}$ (by $35 \%$, note the different scales), being expected, as the top surface is directly heated. The abrupt transition at $3 \mathrm{~s}$ has the same origin, as one of the jumps seen in Fig. 3(a), and after it, we find that (1) $T_{\text {bot }}^{*}$ drops in a manner consistent with heating since the power 


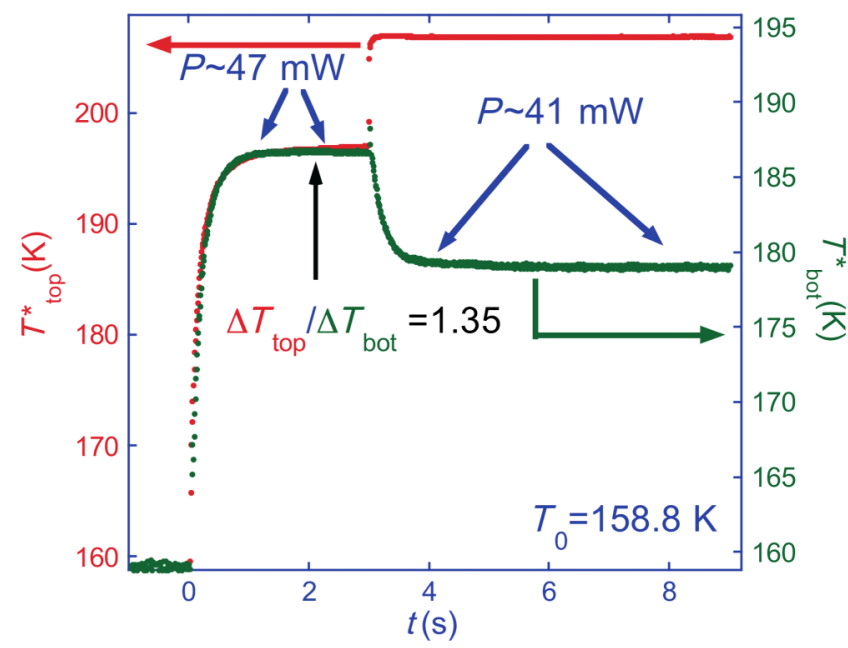

FIG. 4. The time dependencies of the response of crystal $A$ after a high current of $30.5 \mathrm{~mA}$ is applied at $t=0$. The temperatures $T_{\text {top }}^{*}$ and $T_{\text {bot }}^{*}$ are derived by matching the measured timedependent $Y_{\text {top }}$ and $Y_{\text {bot }}$ to the corresponding low-current $Y_{\text {top }}(T)$ and $Y_{\text {bot }}(T)$; see Fig. 3(b). These data are scaled to match for $t<3 \mathrm{~s}$, where the responses differ only by a scaling factor. In this scaling regime, the $\Delta T_{\text {top }}^{*}$ is larger (by about 35\%) than $\Delta T_{\text {bot }}^{*}$, and this is consistent with simple heating and the fact that the heat due to the high current is applied to the top surface. For $t>3 \mathrm{~s}$, something else happens-see the text.

dissipation is smaller beyond $3 \mathrm{~s}\left[V_{\text {top }}\right.$ drops at constant $I_{\text {top }}$ in Fig. 3(a)] and (2) the drop has the same thermal time constant of approximately $300 \mathrm{~ms}$ seen in the initial temperature rise below $1 \mathrm{~s}$. Since $T_{\text {top }}^{*}$ shares neither behavior, it implies that the response of the high-currentdensity top surface is not purely heating.

For the XRD experiments, the $\mathrm{COO}$ state is monitored through a series of $1 \mathrm{D}$ longitudinal $\theta-2 \theta$ scans using $\mathrm{x}$ rays at beam line $\mathrm{X} 22 \mathrm{C}$ of the National Synchrotron Light Source (NSLS, Brookhaven). By fitting to a Lorentziansquared line shape, the peak position, intensity, and width are determined for the $(1,1,12)$ Bragg reflection and the $(1 / 4,1 / 4,12)$ superlattice reflection of the COO phase.

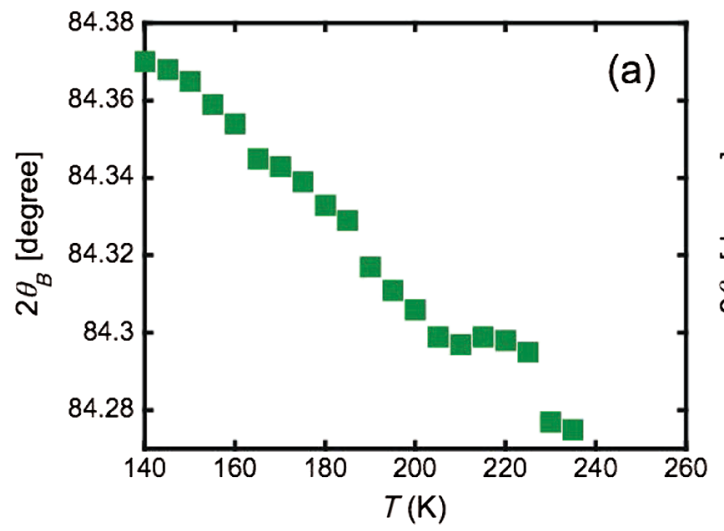

The absence of exchange gas in the cryostat at X22C leads to greater heating effects that necessitate a modified mounting geometry to minimize heating and thus a different crystal $(B)$ to minimize heating. All eight $\mathrm{Au}$ wires from the Au pads of crystal $B$ are clamped between small sapphire pieces and the sapphire substrate that is itself clamped to the cold plate of the Displex cooling stage. We find significant temperature dependence to the lattice constants determined, in this case, by the $(1,1,12)$ Bragg reflection [see Fig. 5(a)]. This thermal expansion acts as an internal, surface-sensitive thermometer that senses the NE surface region since the $\mathrm{x}$-ray penetration depth for $11.2-\mathrm{keV} \times$ rays is $\leq 10 \mu \mathrm{m}<D_{\text {eff }}=40 \mu \mathrm{m}$ in crystal $B$. Thus, to determine $T_{\text {top }}^{*}$ in the surface region with the highest current density, it is only necessary to measure $2 \theta_{B}(I)$, as in Fig. 5(b). Those values of $2 \theta_{B}(I)$ are then converted to $T_{\text {top }}^{*}$ using Fig. 5(a) as the calibration. Some values of $T^{*}$, determined from $2 \theta_{B}(I)$, are shown along the $I(V)$, and they clearly indicate that heating above $T_{\mathrm{COO}}$ cannot cause the vertical region of the $I(V)$.

We now use these $T^{*}$ as a function of power $P=I V$ to construct the expected $I_{h}(V)$ for pure heating. First, $Y_{h}\left(T^{*}\right)$ is found from $T^{*}$ and Fig. 3(b); then, $V_{h}$ is $\sqrt{\left[P / Y_{h}(T *)\right]}$ and $I_{h}=Y_{h}\left(T^{*}\right) V_{h}$. The resulting $I_{h}(V)$ is shown as the circles in Fig. 6. This $I(V)$ is significantly different from that observed experimentally (squares in Fig. 6), thus establishing that a nonequilibrium effect beyond heating gives rise to the behavior. Now, note that the transport $I(V)$ of crystal $B$, shown as the squares in Fig. 6, exhibits an identifiable vertical part qualitatively similar to Fig. 3(a) at $205 \mathrm{~K}$ for crystal $A$. The absence of a jump is likely due to poorer heat removal at $\mathrm{X} 22 \mathrm{C}$, since there is no exchange gas, and as such, the voltage never exceeds that of the vertical part of $I(V)$. In the opposite extreme of fast pulses, heating is negligible, so at short times, the conductance is virtually unchanged from the equilibrium, low-current values. Then in crystal $A$, a much larger conductance jump is required to reach the steady-state vertical portion of the $I(V)$ of Fig. 3(a).

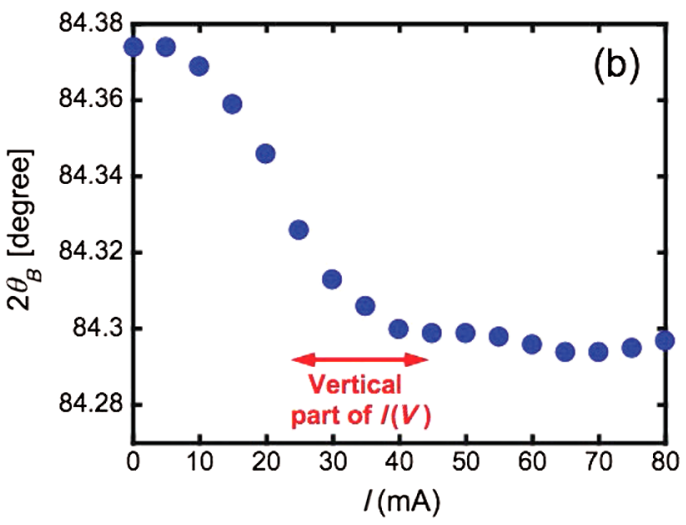

FIG. 5. (a) Bragg reflection $(1,1,12)$ angle $2 \theta$ plotted against temperature $T$ for no current in the crystal. (b) Data for the same reflection plotted versus current at $T_{0}=140 \mathrm{~K}$. 


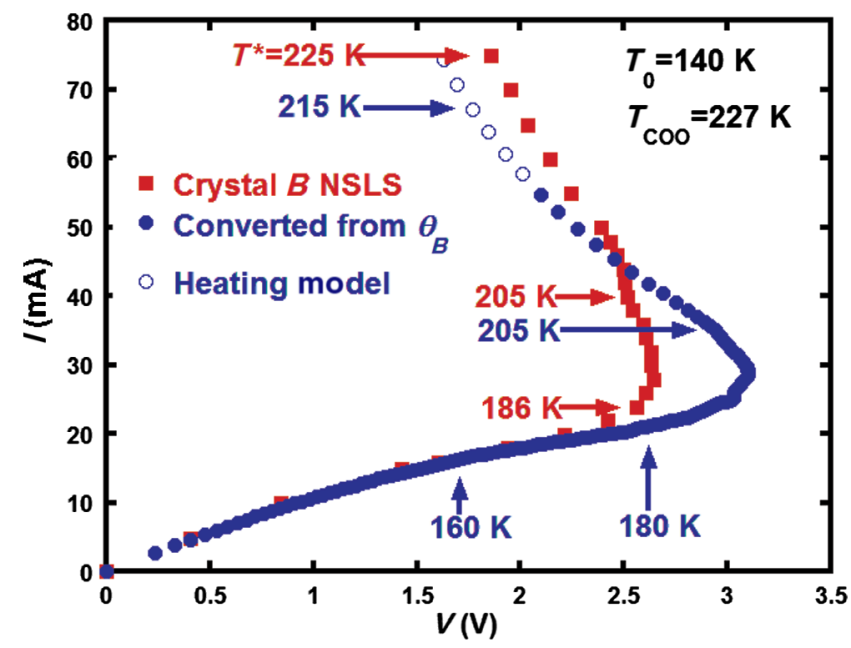

FIG. 6. The current-voltage curve $I(V)$ for crystal $B$ (squares), measured simultaneously with the $(1,1,12)$ Bragg peaks at the NSLS beam line X22C shown in Fig. 5(b). For each $I$ in the above $I(V)$, Fig. $5(\mathrm{~b})$ shows the simultaneously measured $2 \theta_{B}$, which uniquely determines $T^{*}$ [see Fig. 5(a)]. From these data plus the thermal equilibrium transport data of Fig. 3(b), the circles represent the expectation of pure heating, and that clearly disagrees with the experimental $I(V)$ in the vertical region near $2500 \mathrm{mV}$. Note that for $230 \mathrm{~K}>T>212 \mathrm{~K}$, the $2 \theta_{B}$ data of Fig. 5(a) become almost independent of $T$ and thus are not useful as a thermometer. Although this range is not necessary for the conclusions of this paper, we use the measured power $I V$ and a simple heating model to determine the six highest-current, unfilled circles.

The above analyses assume uniform heating within planes perpendicular to the $c$ axis. Nonuniform heating instabilities in the form of a hot channel along the current direction could be consistent with the $Y(T)$ of Fig. 3(b). Because the $\mathrm{x}$-ray beam diameter is greater than the crystal width $w$, a hot channel would lead to broadened satellite reflections for the Bragg and $\mathrm{COO}$ peaks, as they would be effectively constructed from a distribution of peak positions corresponding to the different temperatures. However, we also need to dispel the possibility of multiple hot channels that are each narrow enough for the contribution to the x-ray Bragg peaks to be negligible but conductive enough, by reaching $T \approx T_{\mathrm{COO}}$ with its much higher conductance [see Fig. 3(b)], to emulate the conductance increase in the vertical part of the $I(V)$. Here, we analyze crystal $B$, for which the x-ray-diffraction peak widths are measured as a function of current (Fig. 7). The vertical part of the $I(V)$ of crystal $B$ shown in Fig. 6 is at $V \approx 2500 \mathrm{mV}$ and displays a current increase of approximately $25 \mathrm{~mA}$, so the conductance increase is approximately $0.01 \mathrm{~S}$. In thermal equilibrium, conductance data [Fig. 3(b)] are measured over the length $L$ between voltage contacts and with an effective conducting area of $w D_{\text {eff }}$, where the characteristic length $D_{\text {eff }}$ defines the exponential decay of current density with distance

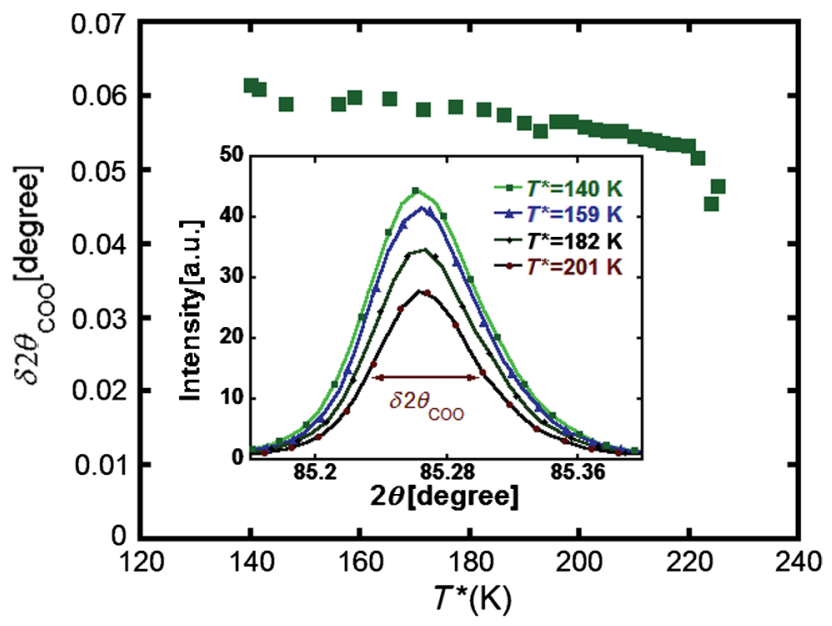

FIG. 7. The full widths of the half maximum of the COO satellite reflections $(1 / 4,1 / 4,12)$ determined as a function of current and plotted against the same $T^{*}$ values used and explained in the caption of Fig. 6. The highest current is $80 \mathrm{~mA}$, so that the vertical part of the $I(V)$ is included. The inset displays some COO peaks for $T^{*}=140,159,182$, and $201 \mathrm{~K}$. For clarity of display, $2 \theta$ of the peak for $T^{*}=159,182$, and $201 \mathrm{~K}$ are shifted to be aligned with that of $T^{*}=140 \mathrm{~K}$.

from the surface, due to crystal anisotropy. For crystal $B$, the conductance at $T_{\mathrm{COO}}$, it is $0.13 \mathrm{~S}$ [Fig. 3(b)]. Then, to emulate the 0.01-S increase in the vertical part of $I(V)$, with channels at $T=T_{\mathrm{COO}}$, would require the total cross-sectional area of all hot channels to be $w D_{\text {eff }} / 13$, since $L$ is unchanged.

If the channels were randomly positioned within the full depth $D_{\text {eff }}$ of the conducting area, their effective width, showing broadened $\mathrm{x}$ rays, would be $w / 13$, and their presence may be difficult to definitively ascertain from the Bragg peaks. However, there are two reasons that make it much more favorable for any hot channels to be at or near the top surface: (1) The current density is the highest there, and (2) the removal of heat from channels at or near the surface is the poorest due to the insulating vacuum interface on one side. Then, it is likely that hot channels would be concentrated at a depth considerably less than both $D_{\text {eff }}$ (approximately 20-40 $\mu \mathrm{m}$ ) and the x-ray penetration depth of $10 \mu \mathrm{m}$, and consequently, their higher surface density would result in an effective width much greater than $w / 13$. Further, it is not realistic to assume an abrupt temperature drop from $T_{\mathrm{COO}}$, in the channel core, to the crystal temperature $T^{*}$ in the neighboring intercore regions. The reason is that the total dissipation within very narrow channels would be small (proportional to the square of their core radius), and thus the thermal gradient outside the channel core could not be large [19]. Although the contributions to the conductance will be small in these neighboring regions, they will considerably increase the effective core size and thus the broadening of the Bragg peak. Therefore, we conclude that the larger lattice 


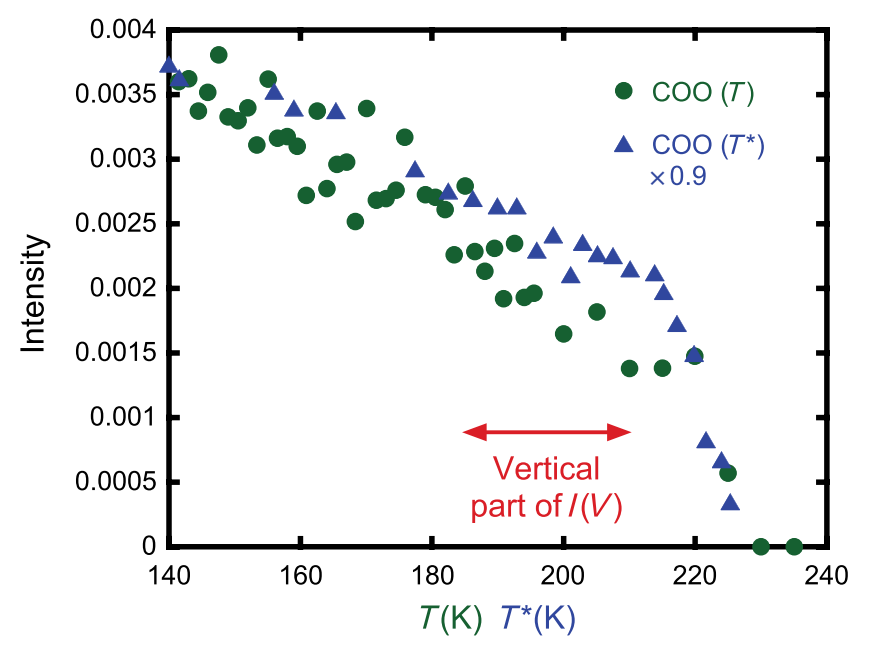

FIG. 8. The integrated intensities of the COO peaks in thermal equilibrium (green circles) are shown for crystal $B$ as a function of $T$. The blue triangles represent states with high current plotted against the same $T^{*}$ values used and explained in the caption of Fig. 6 . The blue triangles have been scaled by a factor of 0.9 , but such a shift is not unusual due to the monitor variation and general drift of the beam line after the ring is shut down and refilled. The five blue triangles with highest $T^{*}$ require the same extrapolation as in Fig. 6. However, these data points are not in the critical vertical part of the $I(V)$ of Fig. 6 and thus are irrelevant to our conclusions.

constants within the hot channels and the neighboring intercore regions would always be readily discernable as a broadening of the diffraction peak. Instead, the COO peak is shown in Fig. 7 to narrow slightly as the current increases through the vertical part of $I(V)$ and up to $80 \mathrm{~mA}$ : The lack of broadening is inconsistent with the nonuniform temperature profile of hot channels.

\section{CHARGE ORDER}

To investigate the possibility of current-induced $\mathrm{COO}$ melting, we use a COO superlattice peak at the $(1 / 4$, $1 / 4,12)$ reflection of crystal $B$. For $I=0$, the integrated intensity of the $\mathrm{COO}$ peak for zero current is shown in Fig. 8 to persist for bath temperatures up to $T_{\mathrm{COO}} \approx 227 \mathrm{~K}$, as expected. When currents are applied, the superlattice peak persists for all effective temperatures $T^{*}$ up to the maximum current of $80 \mathrm{~mA}$ for which $T^{*} \approx 225 \mathrm{~K}$. Then, in vertical portions of the $I(V)$ of Fig. 6, the COO state has not melted and the reflections follow the equilibrium trend versus $T$ (see Fig. 6), albeit at effective temperatures of $T^{*}$. The convincing agreement between the $T$ and $T^{*}$ dependencies implies that $T^{*}$ is a viable effective temperature upon which the COO phase intensity solely depends.

\section{APPLYING THEORY TO EXPERIMENT}

First, the vertical part of the NE experimental $I(V)$ that we observe has been proven to be distinct from the heating reported by Fisher et al. [12], since $T^{*}<T_{\mathrm{COO}}$, and melting of the $\mathrm{COO}$ state reported by Asamitsu et al. [13], since in our case, it has not melted. Having theoretically established that partially charged ODWs are thermodynamically stable, we now address how these ODWs might explain the NE experimental $I(V)$ reported above in terms of a SCDW along these ODWs. Consider the known SCDW materials $\mathrm{NbSe}_{3}$ and $\mathrm{TaS}_{3}$ [14] that display similar threshold electric fields. Any partially charged ODW in a highly anisotropic layered material could emulate these incommensurate, pseudo-1D materials. First, the ODWs contain pseudo-1D charge-density waves, while the effect of commensurability may be softened by fluctuations, since they should introduce elasticity to the long-range charge order given the large periodicity $5<N_{c}<35$. We therefore suggest that a plausible explanation of the NE state we have found in the layered manganite is a SCDW along partially charged 1D ODWs in any of the configurations in Ref. [5] but most likely in the favored one labeled $3 b$ that we use in the above calculations. The desirability of a more direct verification of this conjecture invites further experiments coupled with deeper theoretical constructs.

\section{SUMMARY}

In summary, we predict on theoretical grounds that orbital domain walls, known to exist in the charge and orbital ordered manganites, can be stabilized elastically if they are charged. Then, a sliding charge-density wave along these one-dimensional domain walls presents a potential explanation of the nonequilibrium effect we observe in the bilayer manganite $\operatorname{LaSr}_{2} \mathrm{Mn}_{2} \mathrm{O}_{7}$ : an abrupt transformation into higher-conductance states as high currents are applied.

\section{ACKNOWLEDGMENTS}

The authors would like to acknowledge informative discussions with Peter Littlewood and Ray Osborn and, in particular, with Daniel Khomskii, whose sketch of the ODW orbitals initiated our evaluation of them. This research was funded by the Department of Energy, Office of Basic Energy Sciences, Materials Science and Engineering Division, under Contracts No. DE-AC0206CH11357 at Argonne and No. DE-AC02-98CH10886 at Brookhaven.

[1] J. A. Bert, B. Kalisky, C. Bell, M. Kim, Y. Hikita, H. Y. Hwang, and K. A. Moler, Direct Imaging of the Coexistence of Ferromagnetism and Superconductivity at the $\mathrm{LaAlO}_{3} / \mathrm{SrTiO}_{3}$ Interface, Nat. Phys. 7, 767 (2011).

[2] L. Li, C. Richter, J. Mannhart, and R. C. Ashoori, Coexistence of Magnetic Order and Two-Dimensional Superconductivity at $\mathrm{LaAlO}_{3} / \mathrm{SrTiO}_{3}$ Interfaces, Nat. Phys. 7, 762 (2011). 
[3] J. Zaanen and O. Gunnarsson, Charged Magnetic Domain Lines and the Magnetism of High- $T_{c}$ Oxides, Phys. Rev. B 40, 7391 (1989).

[4] P. G. Radaelli, D. E. Cox, M. Marezio, and S.-W. Cheong, Charge, Orbital, and Magnetic Ordering in $\mathrm{La}_{0.5} \mathrm{Ca}_{0.5} \mathrm{MnO}_{3}$, Phys. Rev. B 55, 3015 (1997).

[5] J. P. Hill, C. S. Nelson, M. V. Zimmermann, Y.-J. Kim, D. Gibbs, D. Casa, B. Keimer, Y. Murakami, C. Venkataraman, T. Gog, Y. Tomioka, Y. Tokura, V. Kiryukhin, T. Y. Koo, and S.-W. Cheong, Orbital Correlations in Doped Manganites, Appl. Phys. A 73, 723 (2001).

[6] D. I. Khomskii and K. I. Kugel, Elastic Interactions and Superstructures in Manganites and Other Jahn-Teller Systems, Phys. Rev. B 67, 134401 (2003).

[7] J. J. Quinn and K. S. Yi, Solid State Physics, Principles and Modern Applications (Springer-Verlag, Berlin, 2009), Chap. 1, p. 30.

[8] J. van den Brink, G. Khaliullin, and D. Khomskii, Charge and Orbital Order in Half-Doped Manganites, Phys. Rev. Lett. 83, 5118 (1999).

[9] D. I. Khomskii and K. I. Kugel, Why Stripes? Spontaneous Formation of Inhomogeneous Structures Due to Elastic Interactions, Europhys. Lett. 55, 208 (2001).

[10] P. M. Chaikin and T. C. Lubensky, Principles of Condensed Matter Physics (Cambridge University Press, Cambridge, England, 1995), Chap. 4, p. 177.

[11] The stability criterion of Ref. [6] relies on the per-site energy and thus does not include the thermodynamically stable ODWs. Because each plaquette contains approximately 1500 sites [5] and their ODWs will contain, at most, with $N_{c}=5$, about 30 charged sites (that are each shared equally with a neighboring plaquette), any correction to the energies used in the stability criterion is likely to be very small.

[12] B. Fisher, J. Genossar, L. Patlagan, and G. M. Reisner, Sliding Charge Density Waves in Manganites?, J. Magn. Magn. Mater. 322, 1239 (2010).

[13] A. Asamitsu, Y. Tomioka, H. Kuwahara, and Y. Tokura, Current Switching of Resistive States in Magnetoresistive Manganites, Nature (London) 388, 50 (1997).
[14] P. A. Lee and T. M. Rice, Current Switching of Resistive States in Magnetoresistive Manganites, Phys. Rev. B 19, 3970 (1979); P. Monceau, N. P. Ong, A. M. Portis, A. Meerschaut, and J. Rouxel, Electric Field Breakdown of Charge-Density-Wave-Induced Anomalies in $\mathbf{N b S e}_{3}$, Phys. Rev. Lett. 37, 602 (1976); A. Zettl, G. Grüner, and A. H. Thompson, Charge-Density-Wave Transport in Orthorhombic $\mathbf{T a S}_{3}$. I. Nonlinear Conductivity, Phys. Rev. B 26, 5760 (1982).

[15] S. Cox, J. Singleton, R. D. McDonald, A. Migliori, and P. B. Littlewood, Sliding Charge-Density Wave in Manganites, Nat. Mater. 7, 25 (2008).

[16] Q. Li, K. E. Gray, H. Zheng, H. Claus, S. Rosenkranz, S. Nyborg Ancona, R. Osborn, J. F. Mitchell, Y. Chen, and J. W. Lynn, Reentrant Orbital Order and the True Ground State of $\mathrm{LaSr}_{2} \mathrm{Mn}_{2} \mathrm{O}_{7}$, Phys. Rev. Lett. 98, 167201 (2007).

[17] T. Kimura, R. Kumai, Y. Tokura, J. Q. Li, and Y. Matsui, Successive Structural Transitions Coupled with Magnetotransport Properties in $\mathrm{LaSr}_{2} \mathrm{Mn}_{2} \mathrm{O}_{7}$, Phys. Rev. B 58, 11081 (1998).

[18] R. Busch, G. Ries, H. Werthner, G. Kreiselmeyer, and G. Saemann-Ischenko, New Aspects of the Mixed State from Six-Terminal Measurements on $\mathrm{Bi}_{2} \mathrm{Sr}_{2} \mathrm{CaCu}_{2} \mathrm{O}_{x}$ Single Crystals, Phys. Rev. Lett. 69, 522 (1992).

[19] Note that nanometer-size channels with small cylindrical core radii $\left(\rho_{0}\right)$ would not alter our conclusion: Their smaller dissipation, approximately $\rho_{0}^{2}$, would lead to a smaller temperature gradient falling away from the "core" at $T_{\mathrm{COO}}$ and thus a much larger effective size than $\rho_{0}$, in which $T$ is well above the background $T^{*}$. There would also be a large number of channels needed $w D_{\text {eff }} /\left(13 \pi \rho_{0}^{2}\right) \approx 730 \mu \mathrm{m}^{2} / \rho_{0}^{2}$, and the distance between them would be $\sqrt{13 \pi} \rho_{0} \approx 6.4 \rho_{0}$, making a significant drop in $T$ between them hard to imagine. The significant propensity for them to be located near the surface would make the distance between them even smaller. 